\title{
CLUSTER VALUES OF ANALYTIC FUNCTIONS ALONG PATHS
}

\author{
T. W. GAMELIN ${ }^{1}$ and MIKIO NIIMURA
}

\section{Summary.}

Let $D$ be a bounded open subset of the complex plane. We consider the cluster values of a meromorphic function $f$ with respect to a family $\Phi$ of paths in $D$ terminating at $\partial D$. Under certain conditions on $\Phi$, it is proved that $\mathrm{Cl}_{\Phi}(f, p)$ contains the boundary of the cluster set of $f$ at a given point $p \in \partial D$, and that every value in $\mathrm{Cl}(f, p) \backslash \mathrm{Cl}_{\Phi}(f, p)$ is assumed by $f$ infinitely often in each neighborhood of $p$ with the possible exception of a set of values of logarithmic capacity zero. The theorems extend results obtained by K. Noshiro [6] for the open unit disc.

\section{Introduction.}

Let $D$ be a domain in the complex plane $C$. We will consider paths in $D$ that terminate at $\partial D$. Such a path $\varphi$ will always be parametrized so that $\varphi(t) \in D$ for $0 \leqq t<1$, while $\varphi(1) \in \partial D$ is the terminal point of $\varphi$.

The cluster set of an extended complex-valued function $f$ along $\varphi$ is denoted by $\mathrm{Cl}(f, \varphi)$. It is the set of limit points in the extended complex plane of $f(\varphi(t))$, as $t \uparrow 1$. For a family $\Phi$ of paths, the $\Phi$-cluster set of $f$ at a point $p \in \partial D$ is defined to be

$$
\mathrm{Cl}_{\Phi}(f, p)=\bigcap_{\varepsilon>0} \overline{\cup\{\mathrm{Cl}(f, \varphi) ; \varphi \in \Phi,|\varphi(1)-p|<\varepsilon\}},
$$

where the closure is taken in the extended complex plane. The full cluster set of $f$ at $p$ is defined as usual to be

$$
\mathrm{Cl}(f, p)=\bigcap_{\varepsilon>0} \overline{f(D \cap \Delta(p ; \varepsilon))},
$$

where $\Delta(p ; \varepsilon)$ is the open disc centered at $p$ with radius $\varepsilon$. Evidently $\mathrm{Cl}_{\Phi}(f, p)$ is a closed subset of $\mathrm{Cl}(f, p)$. We will also be dealing with the range $R(f, p)$ of $f$ at $p$, which consists of those values assumed infinitely often by $f$ in each neighborhood of $p$ :

1 Supported in part by an NSF grant.

Received October 31, 1977. 


$$
R(f, p)=\bigcap_{\varepsilon>0} f(D \cap \Delta(p ; \varepsilon)) .
$$

We consider the problem of imposing conditions on $D$ and $\Phi$ so that every meromorphic function $f$ on $D$ has the following properties.

(1.1) $\quad \mathrm{Cl}_{\Phi}(f, p)$ includes the topological boundary of $\mathrm{Cl}(f, p)$.

(1.2) $\quad \mathrm{Cl}(f, p) \backslash\left[\mathrm{Cl}_{\Phi}(f, p) \cup R(f, p)\right]$ has logarithmic capacity zero.

K. Noshiro $[6 ; 5$, p. 40 , Theorem $9 ; 5$, p. 43 , Theorem 11$]$ has proved that any meromorphic function $f$ on the open unit disc $\Delta$ has properties (1.1) and (1.2), providing the terminal points on $\partial \Delta$ of the family $\Phi$ form a set of full arc-length measure.

With the aid of a theorem from [4] and Bagemihl's theorem on ambiguous points [1], we aim to extend the Noshiro theorems to more general domains. We give two extensions. The first, in Section 3, is valid for arbitrary domains. The second, Section 4, is valid for domains with the property that harmonic measure is carried by an at most countable number of boundary continua. Section 5 includes an example that shows that the family of paths required for this special class of domains may be much smaller than the family required by the results for general domains.

\section{Homotopic paths terminating in $\partial D$.}

In this section, we discuss some background material on homotopy classes of paths in $D$ that terminate at $\partial D$, where $D$ is a bounded domain in the complex plane.

Let $\varphi$ and $\psi$ be two paths in $D$ terminating at $\partial D$. We say that $\varphi$ is homotopic to $\psi$, written $\varphi \simeq \psi$, if there is a continuous family of paths $\varphi_{s}, 0 \leqq s \leqq 1$, in $D$, each terminating at the same point of $\partial D$, such that $\varphi_{0}=\varphi$ and $\varphi_{1}=\psi$. In particular, homotopic paths have the same terminal point.

Let $\pi: \Delta \rightarrow D$ be the universal covering map of $\Delta$ onto $D$. Let $\varrho_{0}$ denote the radial path in $\Delta$ terminating at $e^{i \theta} \varepsilon \partial \Delta$, defined by $\varrho_{\theta}(r)=r e^{i \theta}, 0 \leqq r \leqq 1$. The path $\pi \circ \varrho_{\theta}$ in $D$ is called a conformal ray in $D$. By Fatou's Theorem, the conformal ray $\pi \circ \varrho_{\theta}$ terminates at a point of $\partial D$ for almost all $(d \theta)$ values of the parameter $\theta$. Let $\gamma_{\theta}$ denote the path $\pi \circ \varrho_{\theta}$ whenever it terminates at a point of $\partial D$.

Lemma 1. If $\varphi$ is a path in $D$ terminating at a point of $\partial D$, then any (continuous) lift $\pi^{-1} \circ \varphi$ of $\varphi$ to $\Delta$ is a path in $\Delta$ that terminates at a point of $\partial \Delta$.

Proof. Since $\pi$ is a covering map, the lifted values $\left(\pi^{-1} \circ \varphi\right)(s)$ accumulate towards $\partial \dot{\Delta}$ as $s \uparrow 1$. Suppose that $\pi^{-1} \circ \varphi$ does not terminate at a point of $\partial \Delta$. 
Then there is an open arc of $\partial \Delta$ included in the cluster set $\pi^{-1} \circ \varphi$. Furthermore, the radial cluster set of $\pi$ at each point of this arc includes the value $p$. By Fatou's Theorem, $\pi$ is constant. This contradiction establishes the lemma.

Lemma 2. Let $\varphi_{s}, 0 \leqq s \leqq 1$, be a homotopy of paths in D terminating at $p \in \partial D$. Then there is a lift of the homotopy to paths $\pi^{-1} \circ \varphi_{s}$ in $\Delta$, each terminating at $\partial \Delta$. For any such lift, each path $\pi^{-1} \circ \varphi_{s}$ terminates at the same point of $\partial \Delta$.

Proof. Since $\pi$ is a covering map, we can lift to $\Delta$ the piece of the homotopy $\varphi_{s}(t)$ for parameter values $0 \leqq s \leqq 1$ and $0 \leqq t<1$, to obtain a continuous family $\pi^{-1} \circ \varphi_{s}$ of paths in $\Delta$. By Lemma 1 , each of these paths terminates at a point of $\partial \Delta$. To prove the lemma, it suffices to show that the diameters of the sets

$$
E_{t}=\left\{\left(\pi^{-1} \circ \varphi_{s}\right)(t) ; 0 \leqq s \leqq 1\right\}
$$

tend to zero as $t \uparrow 1$.

Suppose not. Then there is a sequence $t_{n} \uparrow 1$ such that the continua $E_{t_{n}}$ converge to a subset of $\partial \Delta$ including an arc with nonempty interior. Again $p$ belongs to the radial cluster set of $\pi$ at each point of the arc, and this leads to a contradiction.

Lemma 3. If $\varphi$ is a path in $D$ terminating at $p \in \partial D$, then there is a conformal ray $\gamma_{\theta}$ that is homotopic to $\varphi$.

Proof. Suppose that the lift $\pi^{-1} \circ \varphi$ of $\varphi$ to $\Delta$ terminates at $e^{i \theta} \in \partial \Delta$. We claim that $\varphi \simeq \gamma_{\theta}$. We assume for convenience that $\pi^{-1} \circ \varphi$ has initial point 0 , as does the ray $\varrho_{\theta}$.

By Lindelöfs Theorem, $\pi$ has nontangential limit value $p$ at $e^{i \theta}$, so that $\gamma_{\theta}$ terminates at $p$. There is then a domain $U$ in $\Delta$ whose boundary $\partial U$ is a simple closed Jordan curve in $\Delta \cup\left\{e^{i \theta}\right\}$, such that both $\pi^{-1} \circ \varphi$ and the radial segment $\varrho_{\theta}$ are included in $\bar{U}$, while $\pi(z) \rightarrow p$ as $z$ tends to $e^{i \theta}$ through $\partial U$. Such a domain can be constructed, for instance, by covering $\Delta$ by a grid of squares so that the oscillation of $\pi$ on the individual squares tends to zero as the squares approach the boundary $\partial \Delta$ of $\Delta$. By traversing appropriately the outer boundary of the collection of squares in the grid that meet one of the two paths above, one obtains the curve $\partial U$.

Another application of Lindelöfs Theorem shows that $\pi(z) \rightarrow p$ as $z$ tends to $e^{i \theta}$ through $U$. Hence $\pi$ extends continuously to $\bar{U}$, mapping $\bar{U}$ into $D \cup\{p\}$. Since $U$ is topologically a closed disc, it is a simple matter to find a homotopy of the paths $\pi^{-1} \circ \varphi$ and $\varrho_{\theta}$ through paths in $U$ terminating at $e^{i \theta}$. The projection of this homotopy is then a homotopy of $\varphi$ and $\gamma_{\theta}$ through paths in $D$ terminating at $p$. 
LEMMA 4. Let $T$ be a covering transformation of $\Delta$ associated with $\pi$, so that $\pi \circ T=\pi$. If $\gamma_{\theta}$ is any conformal ray that terminates at $p \in \partial D$, and $T\left(e^{i \theta}\right)=e^{i \theta^{\prime}}$, then $\gamma_{\theta^{\prime}}$ also terminates at $p$, and $\gamma_{\theta^{\prime}} \simeq \gamma_{\theta^{\prime}}$.

Proof. Let $\psi(r)=T\left(r e^{i \theta}\right), 0 \leqq r \leqq 1$. Then $\psi$ is a lift of $\gamma_{\theta}$, and $\psi$ terminates at $T\left(e^{i \theta}\right)=e^{i \theta^{\prime}}$. By Lemma 3, $\gamma_{\theta} \simeq \gamma_{\theta}$.

LemMa 5. Suppose that $\gamma_{\theta}$ and $\gamma_{\theta^{\prime}}$ both terminate at a point $p$ of $\partial D$, and that $\gamma_{\theta}$ $\simeq \gamma_{\theta^{\prime}}$. Then there is a covering transformation $T$ such that $T\left(e^{i \theta}\right)=e^{i \theta^{\prime}}$.

Proof. Let $\varphi_{s}, 0 \leqq s \leqq 1$, be a continuous family of paths in $D$ terminating at $p \in \partial D$, such that $\varphi_{0}=\gamma_{\theta}$ and $\varphi_{1}=\gamma_{\theta^{\prime}}$. Let $\psi_{s}=\pi^{-1} \circ \varphi_{s}$ be a lift of the homotopy to $\Delta$, normalized so that $\psi_{1}(t)=t e^{i \theta^{\prime}}, 0 \leqq t \leqq 1$. Now $\psi_{0}$ is a lift of $\gamma_{\theta}$, so there is a covering transformation $T$ satisfying $T\left(r e^{i \theta}\right)=\psi_{0}(r), 0 \leqq r<1$. By Lemma $2, \psi_{0}$ and $\psi_{1}$ have the same terminal point, namely, $e^{i \theta^{\prime}}$. Consequently $T\left(e^{i \theta}\right)=e^{i \theta^{\prime}}$.

We have proved the following result.

THEOREM 1. Let $D$ be a bounded domain in the complex plane, and let $\pi: \Delta$ $\rightarrow D$ be the universal covering map. Then the set of points on $\partial \Delta$ at which $\pi$ has a radial limit is invariant under the group of covering transformations of $\Delta$. There is a one-to-one correspondence between equivalence classes (modulo the group of covering transformations) of points of $\partial \Delta$ at which $\pi$ has a radial limit, and homotopy classes of curves in $D$ terminating at $\partial D$.

Let $h$ be an arbitrary complex-valued function on $D$. A homotopy class $\Lambda$ of paths in $D$ terminating at $\partial D$ is ambiguous for $h$ if there are two paths $\varphi, \psi \in \Lambda$ such that $\mathrm{Cl}(h, \varphi)$ is disjoint from $\mathrm{Cl}(h, \psi)$. In view of the preceding discussion, we can easily extend Bagemihl's theorem on ambiguous points $[1 ; 5$, p. 39 , Theorem 8] from the unit disc to arbitrary domains as follows.

THEOREM 2. There are at most countably many homotopy classes that are ambiguous for a given complex-valued function $h$ on $D$.

Proof. Let $g$ be an arbitrary complex-valued function on $\Delta$. Bagemihl's theorem asserts that the set $E$ of terminal points of homotopy classes that are ambiguous for $g$ is an at most countable subset of $\partial \Delta$. Let $\Lambda$ be a homotopy class of paths in $D$ that is ambiguous for $h$, and let $\varphi, \psi \in \Lambda$ be as above. Let $\pi^{-1} \circ \varphi$ and $\pi^{-1} \circ \psi$ be lifts of $\varphi$ and $\psi$ that terminate at the same point $q \in \partial \Delta$. Then the cluster sets of $h \circ \pi$ along $\pi^{-1} \circ \varphi$ and $\pi^{-1} \circ \psi$ are disjoint, so that $q$ is an ambiguous point for $g=h \circ \pi$. By Bagemihl's theorem, $q$ is restricted to lie in an at most countable subset of $\partial \Delta$. In view of Theorem 1 , there can be at most countably many such homotopy classes $A$. 


\section{A cluster value theorem for arbitrary open sets.}

Let $D$ be a bounded open subset of $C$, with constituent components $D_{1}, D_{2}, \ldots$ By harmonic measure for $D$ we mean the measure $\mu=\sum \mu_{j} / 2^{j}$, where $\mu_{j}$ is the harmonic measure on $\partial D_{j}$ for any point of $D_{j}$. We are interested only in the class of mutual absolute continuity of $\mu$.

Let $f$ belong to $H^{\infty}(D)$, the algebra of bounded analytic functions on $D$. Associated with a subset $Q$ of $\partial D$ there is the essential cluster set of $f$ along conformal rays terminating in $Q$, denoted by $\mathrm{Cl}_{\Gamma}(f, Q)[2,3]$. By definition, this consists of those complex numbers $w$ with the following property: For each $\varepsilon$ $>0$, there is a domain $D_{j}$ and a family of conformal rays in $D_{j}$ of positive measure with respect to the parameter $\theta$, each of which terminates in a point of $Q$, and along each of which $f$ has a limit lying in $\Delta(w ; \varepsilon)$. For $p \in \partial D$, define

$$
\mathrm{Cl}_{\Gamma}(f, p)=\bigcap_{r>0} \mathrm{Cl}_{\Gamma}(f, \Delta(p ; r) \cap \partial D) .
$$

Evidently $\mathrm{Cl}_{\Gamma}(f, p)$ is a closed subset of $\mathrm{Cl}(f, p)$. Furthermore, $\mathrm{Cl}_{\Gamma}(f, p)$ is nonempty if and only if $p$ belongs to the closed support of harmonic measure on $\partial D$.

The cluster set $\mathrm{Cl}_{\Gamma}(f, p)$ has an abstract formulation $[2,3]$. Let $\lambda$ denote the harmonic measure on the maximal ideal space $\mathscr{M}(D)$ of $H^{\infty}(D)$, with closed support denoted by supp $(\lambda)$, and let $\mathscr{M}_{p}(D)$ denote the fiber of $\mathscr{M}(D)$ over $p$. Then $\mathrm{Cl}_{\Gamma}(f, p)$ coincides with the range of (the Gelfand transform of) $f$ on $\mathscr{M}_{p}(D) \cap \operatorname{supp}(\lambda)$. The cluster set $\mathrm{Cl}(f, p)$ coincides with the range of $f$ on $\mathscr{M}_{p}(D)$. Now $\mathscr{M}_{p}(D)$ is the maximal ideal space of the fiber algebra, the restriction of $H^{\infty}(D)$ to $\mathscr{M}_{p}(D)$, while $\mathscr{M}_{p}(D) \cap \operatorname{supp}(\lambda)$ includes the Shilov boundary of the fiber algebra whenever $p \in \operatorname{supp}(\mu)$. An elementary principle of Banach algebra theory then implies that $\mathrm{Cl}_{\Gamma}(f, p)$ includes the topological boundary of $\mathrm{Cl}(f, p)$, at least when $p \in \operatorname{supp}(\mu)$. It is proved furthermore in [4] that $\mathrm{Cl}(f, p) \backslash\left[\mathrm{Cl}_{\Gamma}(f, p) \cup R(f, p)\right]$ has zero logarithmic capacity. Thus to establish (1.1) and (1.2) for a bounded analytic function $f$, it suffices to establish the following:

$$
\mathrm{Cl}_{\Phi}(f, p) \supset \mathrm{Cl}_{\Gamma}(f, p)
$$

We will direct our attention towards formulating conditions which guarantee that (3.1) is valid. The discussion in Section 2 leads naturally to the set of conditions given by the following lemma.

Lemma 6. Let $\Phi$ be a family of paths in D terminating at $\partial D$. Suppose that the family of conformal rays $\gamma_{\theta}$ homotopic to a path in $\Phi$ forms a set of full measure with respect to the parameter $\theta$. Then $\mathrm{Cl}_{\Phi}(f, p) \supset \mathrm{Cl}_{\Gamma}(f, p)$ for each $p \in \partial D$ and each bounded analytic function $f$ on $D$. 
Proof. For convenience, we assume that $D$ is connected, and we carry over the notation of Section 2.

In view of Theorems 1 and 2, there is a set $S$ of parameter values of full arclength measure with the property that for each $\theta \in S$, there exists a path $\varphi \in \Phi$ such that $\varphi \simeq \gamma_{\theta}, f$ has a limit along $\gamma_{\theta}$, and that limit belongs to $\mathrm{Cl}(f, \varphi)$. The defintions of $\mathrm{Cl}_{\Gamma}(f, p)$ and $\mathrm{Cl}_{\Phi}(f, p)$ now immediately yield the conclusion (3.1).

THEOREM 3. Let $D$ be a bounded open subset of $C$, and let $p \in \partial D$ belong to the closed support of harmonic measure. Let $\varepsilon>0$, and let $E$ be a Borel subset of $\partial D$ of harmonic measure zero. Let $\Phi$ be a family of paths in $D$ terminating at $\partial D$ such that each path $\psi$ in $D$ terminating at a point of $[\Delta(p ; \varepsilon) \cap \partial D] \backslash E$ is homotopic to some $\varphi \in \Phi$. Then for each meromorphic function $f$ on $D$,

$$
\mathrm{Cl}_{\phi}(f, p) \text { includes the boundary of } \mathrm{Cl}(f, p) \text {, and }
$$

$$
\mathrm{Cl}(f, p) \backslash\left[\mathrm{Cl}_{\Phi}(f, p) \cup R(f, p)\right] \text { has zero logarithmic capacity. }
$$

Proof. First, assume that $f \in H^{\infty}(D)$. In view of our earlier remarks, it suffices to establish (3.1).

Observe that the family of paths in $D$ terminating at points of $E$ corresponds to a set of parameter values of arc-length measure zero. This is proved, for instance, in [2]. In fact, if $D$ is connected, and if $\tilde{\pi}$ is the nontangential boundary value function associated with the universal covering map $\pi: \Delta \rightarrow D$, then $\tilde{\pi}$ carries the measure $d \theta / 2 \pi$ on $\partial \Delta$ onto the harmonic measure for $\pi(0)$ on $\partial D$.

Now we can adjoin to $\Phi$ all paths in $D$ terminating at points of $(\partial D) \backslash \Delta(p ; \varepsilon)$ without enlarging $\mathrm{Cl}_{\phi}(f, p)$. Once this is done, (3.1) follows immediately from Lemma 6, and the theorem is established for functions $f$ in $H^{\infty}(D)$.

Assume next that $f$ is meromorphic, and that $\mathrm{Cl}(f, p)$ omits a disc $\Delta(w ; \varepsilon)$. Then for $r>0$ sufficiently small, $1 /(f-w)$ is bounded on $D \cap \Delta(p ; r)$. Applying the statement already proved to the bounded analytic function $1 /(f-w)$ on $D \cap \Delta(p ; r)$, we find again that (i) and (ii) hold. (It is at this point that it becomes essential to consider bounded open sets rather than domains, since $D \cap \Delta(p ; \varepsilon)$ need not be connected.)

Finally, assume that $f$ is meromorphic, and that $\mathrm{Cl}(f, p)$ coincides with the extended complex plane $\mathrm{C}^{*}$. In this case, the boundary of $\mathrm{Cl}(f, p)$ is empty, so that (i) is trivial. We must establish (ii).

Suppose that $0 \in \mathrm{Cl}(f, p) \backslash \mathrm{Cl}_{\phi}(f, p)$. Choose $\delta>0$ and $\beta>0$ such that

$$
\liminf _{t \rightarrow 1}|f(\varphi(t))|>\beta
$$

for all $\varphi \in \Phi$ terminating at a point of $\Delta(p ; 2 \delta)$. It suffices to show that $\{|w|<\beta\} \backslash R(f, p)$ has logarithmic capacity zero. 
Let $V$ be the open subset of $D$ defined by

$$
V=\{z \in D ;|z-p|<\delta,|f(z)|<\beta\} .
$$

We claim that $\partial V \cap \partial D$ has zero harmonic measure for $V$. Indeed, suppose not. Let $V_{0}$ be a component of $V$ such that $\partial V_{0} \cap \partial D$ has positive harmonic measure for $V_{0}$. There is then a set $\Omega$ of conformal rays in $V_{0}$ corresponding to parameter values of positive arc-length measure, so that the set $S$ of terminal points of the rays in $\Omega$ lies within $\partial V_{0} \cap \partial D$. In particular, $S$ has positive harmonic measure for $V_{0}$, hence $S$ has positive harmonic measure for $D$. Consider the homotopy classes in $D$ of the rays in $\Omega$ that terminate at points of $S \backslash E$. Each of these homotopy classes includes a path in $\Phi$, and hence is ambiguous for $f$. By Theorem 2, there are at most countably many of these homotopy classes, so that $S \backslash E$ is at most countable. Since $E$ has zero harmonic measure for $D$, also $S$ has zero harmonic measure for $D$. This contradiction establishes our claim.

Now $\partial V$ consists of an at most countable collection of analytic arcs, together with a subset $\partial V \cap \partial D$ which is of zero harmonic measure for $V$. Since $\mathrm{Cl}(f, p)$ $=C^{*}$, there are points of $C \backslash \bar{V}$ arbitrarily near $p$, and $p$ belongs to the closed support of harmonic measure for $V$. Furthermore, $f \in H^{\infty}(V)$ satisfies $|f|=\beta$ on the analytic arcs in $\Delta(p ; \delta) \cap \partial V$. Applying the part of the theorem already proved to the restriction of $f$ to $V$ and to any family of paths in $V$ whose terminal points coincide with the analytic arcs in $\partial V$, we find that the boundary of the cluster set of $\left.f\right|_{V}$ at $p$ is included in the circle $\{|w|=\beta\}$. Now $\mathrm{Cl}(f, p)$ includes $\{|w|<\beta\}$. Assertion (ii), applied to $\left.f\right|_{V}$, then shows that $\{|w|<\beta\} \backslash R(f, p)$ has logarithmic capacity zero. The proof is complete.

At the final stage of the argument, we could cite Corollary 1.4 of [4] or the arguments of [5], instead of the special case of Theorem 3 already proved.

The conclusion (ii) of Theorem 3 is valid even when $p \in \partial D$ does not belong to the closed support of harmonic measure, while the conclusion (i) fails only in a very trivial special case. Indeed, if $p \notin \operatorname{supp}(\mu)$, then there exists $\delta>0$ such that $\Delta(p ; \delta) \backslash D$ has logarithmic capacity zero. There are two possibilities for a meromorphic function $f$ on $D$. The first is that $\mathrm{Cl}(f, p)$ coincides with the extended complex plane $C^{*}$, in which case $C^{*} \backslash R(f, p)$ has logarithmic capacity zero. The second is that $f$ extends to be meromorphic in a neighborhood of $p$, in which case $\mathrm{Cl}(f, p)$ is a singleton. This dichotomy can be established by an elementary argument, as in [5, p. 9, Theorems 1 and 2]. It also follows from the proof given above. 


\section{A special class of domains.}

Fix a domain $D$, and let $\varphi$ and $\psi$ be two paths in $D$ that terminate at $\partial D$. We say that $\varphi$ is weakly equivalent to $\psi$ if $\varphi$ and $\psi$ terminate at the same point $p$, and if for each $\varepsilon>0, \varphi(t)$ and $\psi(t)$ eventually lie in the same component of $D \cap \Delta(p ; \varepsilon)$. This is evidently an equivalence relation, leading to weak equivalence classes of paths.

It is not hard to establish that if $\varphi$ and $\psi$ both terminate at the same point of $\partial D$, then $\varphi$ is not weakly equivalent to $\psi$ if and only if there exists $\varepsilon>0$ and a continuum $K$ in $\overline{\Delta(p ; \varepsilon)} \cap \partial D$ such that $p \in K$, and $\varphi(t)$ and $\psi(t)$ eventually lie in different components of $\Delta(p ; \varepsilon) \backslash K$. Homotopic paths are weakly equivalent, but weakly equivalent paths need not be homotopic.

Let $h$ be any complex-valued function on $D$. A weak equivalence class of paths is ambiguous for $h$ if there are paths $\varphi$ and $\psi$ in the equivalence class such that $\mathrm{Cl}(h, \varphi)$ is disjoint from $\mathrm{Cl}(h, \psi)$.

Consider first these definitions in the case of the open unit disc $\Delta$. Any two paths in $\Delta$ terminating at the same point of $\partial \Delta$ are weakly equivalent, and in fact homotopic. Hence the weak equivalence classes and the homotopy classes coincide, and these are in one-to-one correspondence with points of $\partial \Delta$. Bagemihl's Theorem asserts that there are at most countably many weak equivalence classes that are ambiguous for a given function $h$.

Next consider the case in which $D$ is a simply connected domain, not the entire complex plane. Then any two weakly equivalent paths are easily seen to be homotopic, so that again the weak equivalence classes coincide with the homotopy classes. Furthermore, the results of Section 2 show that there is a one-to-one correspondence between homotopy classes of paths in $D$ terminating at $\partial D$, and points of $\partial \Delta$ at which the conformal map $\pi: \Delta \rightarrow D$ has a radial limit. Each homotopy class of paths contains precisely one path of the form $\pi\left(r e^{i \theta}\right), 0 \leqq r \leqq 1$, where $\pi\left(e^{i \theta}\right)$ is the radial limit of $\pi$ at $e^{i \theta}$.

Let $h$ be a complex-valued function on the simply connected domain $D$. Consider an ambiguous weak equivalence class $\Lambda$ for $h$, and let $\varphi$ and $\psi$ be two paths in the class for which $\mathrm{Cl}(h, \varphi)$ is disjoint from $\mathrm{Cl}(h, \psi)$. Now $\Lambda$ is specified uniquely by the terminal point $e^{i \theta}$ of the paths $\pi^{-1} \circ \varphi$ and $\pi^{-1} \circ \psi$ in $\Delta$. Since the cluster sets of $h \circ \pi$ along the paths $\pi^{-1} \circ \varphi$ and $\pi^{-1} \circ \psi$ are disjoint, $e^{i \theta}$ is an ambiguous point for $h \circ \pi$. By Bagemihl's theorem there are at most countably many such points. Consequently there are at most countably many weak equivalence classes of paths in $D$ that are ambiguous for $h$.

Now we extend Bagemihl's theorem one step further.

TheOREM 4. Let $D$ be a domain in the complex plane, and let $K$ be a connected component of $\partial D$ containing more than one point. Let $h$ be an arbitrary complex- 
valued function on $D$. Then there are at most countably many weak equivalence classes with terminal points in $K$ that are ambiguous for $h$.

Proof. Each component of the complement $C^{*} \backslash K$ of $K$ in the extended complex plane $C^{*}$ is simply connected. We apply the preceding discussion (as we may) to the component $V$ of $C^{*} \backslash K$ containing $D$, and to any extension of $h$ to $V$.

Let $\varphi$ and $\psi$ be paths in $D$ terminating at $p \in K$. If $\varphi$ and $\psi$ are weakly equivalent in $D$, they are certainly weakly equivalent in $V$. We claim that the converse is also true. Indeed, suppose $\varphi$ and $\psi$ are not weakly equivalent in $D$. Then there exists $\varepsilon>0$ and a continuum $Q \subset \partial D$ such that $p \in Q$, and $\varphi(t)$ and $\psi(t)$ eventually lie in different components of $\Delta(p ; \varepsilon) \backslash Q$. Now $Q \subset K$, so that $\varphi(t)$ and $\psi(t)$ eventually lie in different components of $\Delta(p ; \varepsilon) \backslash K$. Hence $\varphi$ and $\psi$ are not weakly equivalent in $V$.

Thus the weak equivalence classes in $D$ terminating at points of $K$ are in one-to-one correspondence with a certain subset of the weak equivalence classes of $V$. Evidently classes in $D$ that are ambiguous for $h$ correspond to classes in $V$ that are ambiguous for any extension of $h$. The remarks preceding the theorem show that there are at most countably many such classes.

Using Theorem 4, we can now establish the following analogue of Theorem 3.

THEOREM 5. Let $D$ be a bounded open subset of $C$ such that harmonic measure for $D$ is carried by an at most countable number of connected components of $\partial D$. Let $p \in \partial D$ belong to the closed support of harmonic measure. Let $\varepsilon>0$, and let $E$ be a Borel subset of $\partial D$ of harmonic measure zero. Let $\Phi$ be a family of paths in $D$ terminating at $\partial D$ such that each path $\psi$ in $D$ terminating at a point of $[\Delta(p ; \varepsilon) \cap \partial D] \backslash E$ is weakly equivalent to a path in $\Phi$. Then for each meromorphic function $f$ on $D$,

(i) $\mathrm{Cl}_{\Phi}(f, p)$ includes the boundary of $\mathrm{Cl}(f, p)$, and

(ii) $\mathrm{Cl}(f, p) \backslash\left[\mathrm{Cl}_{\Phi}(f, p) \cup R(f, p)\right]$ has logarithmic capacity zero.

Furthermore, if $f \in H^{\infty}(D)$, then

(iii) $\mathrm{Cl}_{\Phi}(f, p) \supset \mathrm{Cl}_{\Gamma}(f, p)$.

Proof. Again we consider first $f \in H^{\infty}(D)$, in which case it suffices to establish (iii).

Assume for convenience that $D$ is connected.

Let $J$ be an at most countable union of components of $\partial D$ that carries harmonic measure. By Theorem 4, there is an at most countable number of weak equivalence classes of paths that terminate at $J$ and that are ambiguous 
for $f$. By enlarging $E$, we can assume that $E$ includes the terminal points of these ambiguous equivalence classes.

Suppose $w \in \mathrm{Cl}_{\Gamma}(f, p)$. Let $\delta>0$ satisfy $\delta<\varepsilon$. According to the definition, there is a Borel subset $S$ of $\partial \Delta$ of positive length such that for each $e^{i \theta} \in S$, the conformal ray $\gamma_{\theta}$ terminates at a point of $\Delta(p ; \delta) \cap \partial D$, and $f \circ \pi$ has radial limit at $e^{i \theta}$ that belongs to $\Delta(w ; \delta)$. By discarding from $S$ a set of zero length, we can assume that for each $e^{i \theta} \in S$, the conformal ray $\gamma_{\theta}$ terminates at a point of $J \backslash E$. Since $\delta<\varepsilon$, there is for each $e^{i \theta} \in S$ a path $\varphi_{\theta} \in \Phi$ that is weakly equivalent to $\gamma_{\theta}$. Since the weak equivalence class of $\gamma_{\theta}$ is not ambiguous for $f$, the limit of $f$ along $\gamma_{\theta}$ belongs to $\mathrm{Cl}\left(f, \varphi_{\theta}\right)$. In particular, there exists $\varphi \in \Phi$ such that $\mathrm{Cl}(f, \varphi)$ meets $\Delta(w ; \delta)$. Since this is true for all small $\delta>0, w$ belongs to $\mathrm{Cl}_{\Phi}(f, p)$, and (iii) is established, along with (i) and (ii).

Now suppose that $f$ is meromorphic. The case in which $\mathrm{Cl}(f, p) \neq \mathrm{C}^{*}$ can be reduced to the case in which $f$ is bounded, as in the proof of Theorem 3. So we assume that $\mathrm{Cl}(f, p)=\mathrm{C}^{*}$. Then (i) is trivial, and the proof of (ii) proceeds in exactly the same way as the proof of the corresponding assertion of Theorem 3 , except that "homotopy classes" must be replaced by "weak equivalence classes."

\section{An example.}

Let $\left\{\Delta_{n}\right\}$ be a sequence of closed disjoint subdiscs of the open unit disc $\Delta$ such that the $\Delta_{n}$ accumulate on the entire boundary $\partial \Delta$ of $\Delta$, while the radii $r_{n}$ of the $\Delta_{n}$ satisfy $\sum r_{n}<\infty$. Let $D$ be the champagne bubble domain defined by

$$
D=\Delta \backslash \bigcup \Delta_{n} \text {. }
$$

Any two paths in $D$ terminating at the same point of $\partial D$ are weakly equivalent. Furthermore, harmonic measure for $D$ is absolutely continuous with respect to arc length on $\partial D$.

Note that for almost every $e^{i \theta} \in \partial \Delta$, there is a radial interval in $D$ that terminates at $e^{i \theta}$. This is because the discs $\Delta_{n}$ for $n \geqq N$ project radially onto a subset of $\partial \Delta$ of length on the order of $2 \sum_{n=N}^{\infty} r_{n}$, which tends to zero as $N \rightarrow \infty$.

For our family $\Phi$, we consider a family of radial paths in $D$ terminating at $\partial \Delta$ on a set of full arc-length measure, together with paths in $D$ terminating at each $\partial \Delta_{n}$ on a set of full arc-length measure. The family $\Phi$ satisfies the hypotheses of Theorem 5, when $E$ is taken to be the subset of $\partial D$ at which no path in $\Phi$ terminates.

On the other hand, for each $e^{i \theta} \in \partial \Delta$, there is an uncountable number of homotopy classes of paths in $D$ that terminate at $e^{i \theta}$. Consequently the family $\Phi$ falls far from fulfilling the hypotheses of Theorem 3. 


\title{
BIBLIOGRA PHY
}

1. F. Bagemihl, Curvilinear cluster sets of arbitrary functions, Proc. Nat. Acad. Sci. U.S.A. 41 (1955), 379-382.

2. T. W. Gamelin, Lectures on $H^{\infty}(D)$, Notas de Matemática, La Plata, Argentina, 1972.

3. T. W. Gamelin, Iversen's theorem and fiber algebras, Pacific J. Math. 46 (1973), 389-414.

4. T. W. Gamelin, Cluster values of bounded analytic functions, Trans. Amer. Math. Soc. 225 (1977), 295-306.

5. K. Noshiro, Cluster Sets (Ergebnisse Math. Grenzgebiete, N. F. 28), Springer-Verlag, Berlin Göttingen - Heidelberg, 1960.

6. K. Noshiro, Cluster sets of functions meromorphic in the unit circle, Proc. Nat. Acad. Sci. U.S.A. 41 (1955), 398-401.

\author{
DEPARTMENT OF MATHEMATICS \\ UNIVERSITY OF CALIFORNIA \\ LOS ANGELES \\ CALIFORNIA 90024 \\ AND \\ DEPARTMENT OF MATHEMATICS \\ SHIBAURA INSTITUTE OF TECHNOLOGY \\ 3-9-14, SHIBAURA MINATO-KU \\ TOKYO \\ JAPAN
}

